\title{
Scanning Electron Microscopy of the Retinal Arterioles in Normotensive and Spontaneously Hypertensive Rats
}

\author{
Shigeki Takahashi, Tadao Sekino, and Hiroshi Yoshimoto
}

Retinal arterioles of spontaneously hypertensive rats, consisting of 2 rats (4 eyes) of $\mathrm{F}_{21}$ and 4 normotensive rats (8 eyes) were observed with a scanning electron microscope (Nihon-denshi JSM-U3).

The following results were obtained.

1. The luminal surface of retinal arterioles in normotensive rats was uneven along the axis of blood vessels.

2. The luminal surface of retinal arterioles in SHR was more uneven than that in the normotensive rats.

3. At the site of branching of the retinal arterioles, the longitudinal endothelial fold of luminal surface intruded on the endothelium of its stem, while the endothelium at the orifice of that branch bulged markedly into the lumen. 